\title{
GDF15 as a Novel Biomarker for Monitoring Danusertib Activity
}

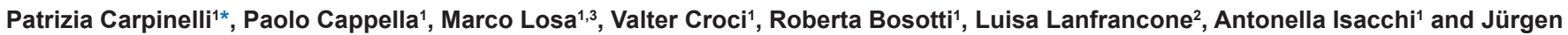
Moll ${ }^{1}$

${ }^{1} \mathrm{BU}$ Oncology, Nerviano Medical Sciences srl, Nerviano (MI), Italy

${ }^{2}$ Department of Experimental Oncology, Istituto Europeo di Oncologia, Milan, Italy

${ }^{3}$ Present Address: Mouse and Animal Pathology Laboratory, Fondazione Filarete, Milan, Italy

\begin{abstract}
Biomarkers that indicate biological activity and/or efficacy are a potentially useful tool in the development of molecularly targeted therapeutics. Most pharmacodynamic assays measure the response to drugs in tissues, a procedure requiring tissue biopsies, which is often not available. In this study, we identify GDF15 as a potential plasma biomarker for Danusertib (formerly PHA-739358), an Aurora kinase inhibitor, currently in phase I/II clinical studies. Cell lysates and cell-free culture supernatants from human tumor isogenic cell lines were assessed for GDF15 levels following treatment with Danusertib, by using Western Blotting and Elisa. GDF15 levels were also measured in plasma from xenografted mice as well as in plasma from patients before and after Danusertib treatment. At the protein level an increased level of GDF15 was found in tissue culture cells after Danusertib treatment and GDF15 expression was also detected in plasma of xenografted mice treated with the compound. Hence, both cellular and xenograft models of human cancer showed a clear correlation with p53 pathway activation. Danusertib treatment also resulted in a significantly increased expression of GDF15 protein levels in the plasma of examined patients, underlining technical feasibility of translating this putative biomarker to clinical studies. Determination of GDF15 levels in plasma is feasible and enabled us to follow the kinetic of Danusertib activity in plasma from cancer xenografted mice and from patients. These findings should allow us to evaluate systematically the kinetics of p53 activation by Danusertib in future clinical studies.
\end{abstract}

Keywords: Danusertib; Plasma biomarker; p53 positive cells

Abbreviations: GDF15: Growth differentiation factor 15; FCS: Fetal Calf Serum; ELISA: Enzyme-Linked Immunosorbent Assay

\section{Introduction}

In clinical practice, biomarkers are widely used for diagnosis, for disease prognosis, and for optimizing therapeutic strategies and the appropriate use of biomarkers can guide the discovery and development of novel therapeutics.

The identification of a suitable and easily measured marker of antitumor activity in the clinical setting can help and accelerate clinical development. The development of microarray technologies for largescale characterization of mRNA expression pattern has made it possible to systematically search for molecular markers which can serve as pharmacodynamic or predictive biomarkers for clinical endpoints [1]. Several studies show that gene expression information generated by microarray analysis of human tumors can predict clinical outcome $[2,3]$. The central goal of our work was the identification of biomarkers whose compound-induced expression provides sufficient information about the mechanism of the compound.

In about half of all human tumors, p53 is not expressed or is mutated, and even when $\mathrm{p} 53$ wild type is expressed, its normal regulatory functions are often compromised by other mechanisms. Restoration of p53 function in established tumors (including lymphomas, sarcomas, and hepatocellular carcinomas) causes tumor regression in vivo and could represent an effective new approach to treat cancer [4,5]. Given the variety of mechanisms by which tumors disrupt p53 pathway function, different approaches can be envisaged to restore $\mathrm{p} 53$ function in diverse settings. Some are already in various stages of preclinical or clinical development like gene therapy approaches directly introducing wild-type p53 genes into tumors or the small molecule PRIMA-1 that has been reported to restore $\mathrm{p} 53$ function in cells bearing $\mathrm{p} 53$ mutations $[6,7]$. Another approach to increase p53 activity is by relieving its inhibition by MDM2 using the small molecule Nutlin [8].
Danusertib, a small-molecule 3-aminopyrazole-derivative with strong activity towards all the Aurora kinase family members causes an Aurora B kinase inhibition-related cellular morphology and molecular mechanism of action upon treatment of cells in vitro and in vivo, as assessed by modulation of phosphorylation of histone $\mathrm{H} 3$ in position serine 10 [9]. The compound is currently tested in clinical phase I/II trials both in solid tumors and hematological malignancies [10-12]. By using a cDNA microarray approach on five different cell lines treated with Danusertib, we previously identified a specific enrichment in p53-dependent genes (Bosotti R. and Carpinelli P., personal communication). Among these genes, expression of GDF15 (Growth Differentiation Factor 15), a secreted protein belonging to transforming growth factor- $\beta$ (TGF- $\beta$ ) superfamily, was induced [13]. GDF15 is also known as placental bone morphogenetic protein, macrophage inhibitory cytokine-1, prostate derived factor, placental TGF- $\beta$ and nonsteroidal anti-inflammatory drug-activated protein 1 [14-17]. It is highly expressed in epidermis in rat embryos and its mRNA is detected in diverse epithelia in adult rat tissues at different levels [16]. GDF15 is generated as a $40-\mathrm{kDa}$ propeptide from which the $\mathrm{N}$ terminus is cleaved and a $30-\mathrm{kDa}$ disulfide-linked dimeric protein is secreted as the active form. It has been implicated in a variety of functions directly related to tumorigenicity including antiproliferative

*Corresponding author: Patrizia Carpinelli, Nerviano Medical Sciences Srl, Viale Pasteur, 10, 20014 Nerviano (MI) Italy, Tel: +39 0331 581532; E-mail: patrizia. carpinelli@nervianoms.com

Received May 26, 2011; Accepted August 25, 2011; Published December 30, 2011

Citation: Carpinelli P, Cappella P, Losa M, Croci V, Bosotti R, et al. (2011) GDF15 as a Novel Biomarker for Monitoring Danusertib Activity. J Mol Biomark Diagn S2:001. doi:10.4172/2155-9929.S2-001

Copyright: (C) 2011 Carpinelli P, et al. This is an open-access article distributed under the terms of the Creative Commons Attribution License, which permits unrestricted use, distribution, and reproduction in any medium, provided the original author and source are credited 
and pro-apoptotic effects $[18,17]$. GDF15 is an important downstream mediator of DNA damage signaling and it has been shown as one of the direct transcriptional targets of p53 $[19,17]$. In addition it is a direct downstream target of TAp63, and TAp63-mediated induction of GDF15 plays an important role during keratinocyte differentiation [20]. Many antitumorigenic compounds, including cyclooxygenase inhibitors, retinoids, genistein, resveratrol, and vitamin $\mathrm{D}$ have been shown to up-regulate GDF15 expression [14,21-24]. Induction of GDF15 can occur via p53-dependent or p53-independent pathways $[25,19,17,26,27]$. Based on our previous finding showing upregulation of GDF15 upon Danusertib treatment (Bosotti R. and Carpinelli P., personal communication), in this study we explored the feasibility to use GDF15 as secreted biomarker to follow the activity of the Aurora kinase inhibitor, Danusertib.

\section{Materials and Methods}

\section{Cell culture}

HCT116 p53 wt and p53 -/- cell lines obtained from Bert Vogelstein [28], were cultured at $37^{\circ} \mathrm{C}$ in $5 \% \mathrm{CO}_{2}$, in McCoy's medium with heat inactivated 10\% Fetal Calf Serum (FCS) and $1 \%$ penicillin/ streptomycin (Sigma). In all the experiments cells were treated with Danusertib at the indicated concentrations and times, starting from a stock solution of $10 \mathrm{mM}$ in DMSO.

\section{Cell cycle analysis}

All of the cells in the plates including cells floating in the medium were collected and analyzed. Adherent cells were released from the plastic by trypsin treatment. Cells were rinsed in PBS and fixed in $70 \%$ ethanol in PBS; then were stained in propidium iodide solution $(25$ $\mu \mathrm{g} / \mathrm{ml}$; Sigma) containing citrate sodium (0.1\%; Sigma), RNase A (5 $\mu \mathrm{g} / \mathrm{ml}$; Sigma), Igepal CA-630 $(0.1 \mu \mathrm{g} / \mathrm{mL}$; Sigma) and incubated for $30 \mathrm{~min}$ at $37^{\circ} \mathrm{C}$. Samples were analyzed by flow cytometry with a BD FACSCalibur $^{\text {Tm }}$ system (Becton Dickinson, Franklin Lakes, NJ). All aggregates were removed during analysis by an appropriate gate on Area/Width parameters and DNA content analysis was performed on $\geq 10,000$ gated cells.

\section{Immunoblotting}

Cells or tissues extracts were prepared in $125 \mathrm{mM}$ Tris- $\mathrm{HCl} \mathrm{pH}$ 6.8 and 2\% SDS. Samples were sonicated and heated for $5 \mathrm{~min}$ at $95^{\circ} \mathrm{C}$. Protein concentration was determined by $\mathrm{BCA}^{\mathrm{m}}$ Protein Assay (Pierce, Rockford, IL). Equal amounts of protein were separated on SDS-polyacrylamide gels and transferred to nitrocellulose membrane (Schleicher \& Schuell). Immunoblot analysis was performed using the following antibodies: anti phospho histone H3 Ser 10 and anti GDF15 (Upstate Biotech, Lake Placid, NY); anti histone H3 (Abcam, Cambrige, UK); anti-p21 (BD Biosciences, Oxford, UK); anti GDF15, anti GAPDH, anti Parp and p53 antibodies were purchased from Santa Cruz Biotechnology, Inc. (Santa Cruz, CA). Immunoblotting was performed according to standard methods. ECL chemiluminescence kit (GE Healthcare Amersham) was used for detection.

\section{ELISA Assay}

Cell-free culture supernatants were centrifuged at $16,000 \mathrm{~g}$ for 5 mins, and frozen at $-80^{\circ} \mathrm{C}$ until assayed. Plasma samples were centrifuged at $1,000 \times \mathrm{g}$ for $15 \mathrm{mins}$ at $4^{\circ} \mathrm{C}$, within $30 \mathrm{mins}$ of collection, and aliquots were stored at $-80^{\circ} \mathrm{C}$ until analysis. The GDF15 ELISA used for quantification of GDF15 in cell culture media and blood samples was performed with Quantikine ELISA kit for human GDF15 (R\&D Systems Inc. MN) following the manufacturer's protocol. The
GDF15 levels are expressed in $\mathrm{pg} / \mathrm{mL}$ of plasma and were measured twice in duplicate.

\section{In vivo experiments}

Athymic Balb nu-nu mice, 5-6 week-old were obtained from Harlan, Italy. All animal studies were carried out in compliance with Italian Legislative Decree N.116 dated January 27, 1992, and the European Communities Council Directive N.86/609/EEC concerning the protection of animals used for experimental or other scientific purposes and according to Institutional Policy Regarding the Care and Use of Laboratory Animals. $2 \times 10^{6}$ HCT116 human colon adenocarcinoma cells (from ATCC) were transplanted s.c. in athymic mice. Mice bearing a palpable tumor $\left(300-400 \mathrm{~mm}^{3}\right)$ were randomized (5 animals/group) and treated with vehicle or a single dose of $40 \mathrm{mg} / \mathrm{kg}$ Danusertib by i.v. route. Tumor and blood samples were collected at 8 , 24 and 48 hours after the treatment and prepared for Western blot and immunohistochemistry analysis as described.

\section{Immunohistochemistry}

Tumors were fixed in $10 \%$ buffered formalin for 24 hours and embedded in paraffin. Immunohistochemistry was done as previously described [29]. Serial sections were incubated with primary antibodies against phospho histone H3 Ser10 (rabbit polyclonal, Upstate; 06-570); p53 (mouse monoclonal, Dako; M7001); p21 (mouse monoclonal, BD Biosciences; 556430) for 1 hour at room temperature. Dako EnVision Labeled Polymer-HRP anti-mouse or ant-rabbit were used as secondary antibodies. All slides were incubated with DAB Substrate Chromgen System (Dako).

\section{Image analysis}

Sections were evaluated in blind by two independent operators using a Zeiss microscope equipped with a charge-coupled device camera (Evolution MP-color, Media Cybernetics). For each biomarker quantification of positive cells was done using Image Pro Plus software (Media Cybernetics). Four random pictures, chosen in viable tumor areas were collected at 200X magnification for each sample, and analyzed. Data are reported as the mean of positive cells counted in the four fields for each sample.

\section{Patient samples}

Following institutional research ethics board approval and informed consent, heparinized plasma samples were obtained in a phase I clinical trial where patients received escalating doses of Danusertib (24-hours infusion every 14 days) for evaluation of pharmacokinetics (PK) of the compound. Plasma samples collected before, during, and after the end of infusion were centrifuged at $1,000 \times \mathrm{g}$ for $15 \mathrm{mins}$ at $4^{\circ} \mathrm{C}$, within 30 mins of collection, and aliquots were stored at $-80^{\circ} \mathrm{C}$ until analysis.

\section{Statistical analysis}

Results of in vitro assays as well as in vivo assays and patient samples are reported as the mean. Statistical significance was assessed by the Mann-Whitney U test using GraphPad Prism software package v.5.02 (GraphPad Software, Inc.). The level of significance was set at P $<0.05$ (in figures, $0.001<^{* *}<0.01$, and $0.01<^{*}<0.05$ ).

\section{Results}

Induction of GDF15 protein upon Danusertib treatment in vitro

In order to characterize specific target genes regulated by Danusertib 
treatment, we have transcriptionally analysed its effects in several cell lines, differing only in p53 status, and have identified a subset of genes specifically modulated by $\mathrm{p} 53$ in response to the compound (Bosotti R. and Carpinelli P., personal communication).

To further validate the microarray gene expression data, the relevance of p53 in response to the Aurora kinase inhibitor, Danusertib, was examined in the isogenic HCT116 cell lines which were either wild type (HCT116 wt) or deleted (HCT116 p53-/-) for p53 [28]. Cell cycle analysis by flow cytometry demonstrated that after $24 \mathrm{hrs}$ of treatment, Danusertib induces the same degree of polyploidy $(>4 \mathrm{~N})$ in both cell lines independently on the p53 status (Figure 1). Following the cell growth after washing-out the compound, in the p53 null cells a relative increase in the polyploidy population appeared in comparison to the wild type p53 expressing cells. In order to distinguish the genes which were regulated by $\mathrm{p} 53$, we previously performed qPCR for selected genes shown to be regulated in the Microarray experiment and determined their protein levels in the HCT116 wt and p53-/- cells (Bosotti R. and Carpinelli P., personal communication). Treatment of exponentially growing HCT116 wt and p53-/- cells with $1 \mu \mathrm{M}$ Danusertib for $24 \mathrm{hrs}$, followed by 24 or $48 \mathrm{hrs}$ wash-out, showed a time-dependent modulation for cellular proteins related to Danusertib mechanism of action, like P-histone $\mathrm{H} 3$ and Parp cleavage, with minor differences between the two cell lines (Figure 2). We also found an upregulation of $\mathrm{p} 53$ that is maintained at the longer time points. The mechanism for this long-lasting activation of p53 is not the result of activation of a DNA damage pathway, but rather appears to involve an Aurora A-dependent p 53 phosphorylation and its dissociation from MDM2, such that p53 is no longer targeted for degradation [30,31].
Since we were particularly interested in the identification of biomarkers for Danusertib activity which can be easily followed in blood, we focused our attention towards GDF15, a secreted protein of the TGF- $\beta$ superfamily (Figure 2). We observed a clear time-dependent induction of this protein for both higher and lower molecular weight forms specifically in HCT116 p53 wt cells (Figure 2).GDF15 is generated as a $40 \mathrm{kDa}$ propeptide from which the $\mathrm{N}$-terminus is cleaved and a 30 $\mathrm{kDa}$ disulfide-linked dimeric protein is secreted as the active form. Thus, we analyzed the effect of Danusertib on the levels of secreted GDF15 in the cell-free culture supernatants of the same cells which were also investigated by Western Blotting. Soluble GDF15 protein could be detected in cell-free culture supernatants by using a sensitive ELISA method. Following Danusertib treatment for $24 \mathrm{hrs}$, the levels of GDF15 secreted protein rise substantially up to $48 \mathrm{hrs}$ after compound wash-out (Figure 3). Interestingly, a minor increase is also observed in the p53 null cells. This might be contributed by a p53 independent pathway mediated by the balance of Sp1 and EGR-1 family members which are reported to regulate GDF15 [32-34].

\section{In vivo modulation of GDF15 by Danusertib}

In order to test if GDF15 modulation can be also followed in vivo, we evaluated the levels of this protein in plasma of HCT116 tumor bearing mice treated either with vehicle or a single dose of Danusertib $(40 \mathrm{mg} / \mathrm{kg})$. Mice were sacrificed and blood and tumors were collected at 8,24 and $48 \mathrm{hrs}$ after the treatment. GDF15 levels increased significantly in plasma beginning after $8 \mathrm{hrs}$ of Danusertib treatment and the effect continued up to $48 \mathrm{hrs}$ (Figure 4A). As the ELISA used was specific for detection of human GDF15, only tumor-derived GDF15 was measured in this study and not the endogenous murine

\section{Time of treatment (hrs)}

6
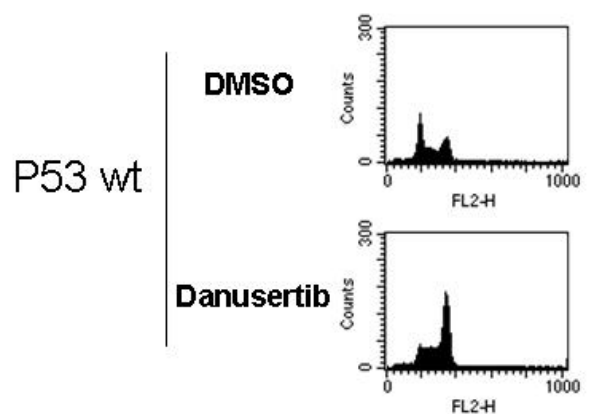

DMSO

P53 -/-

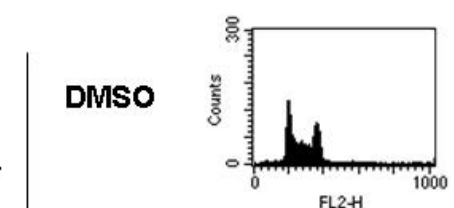

24
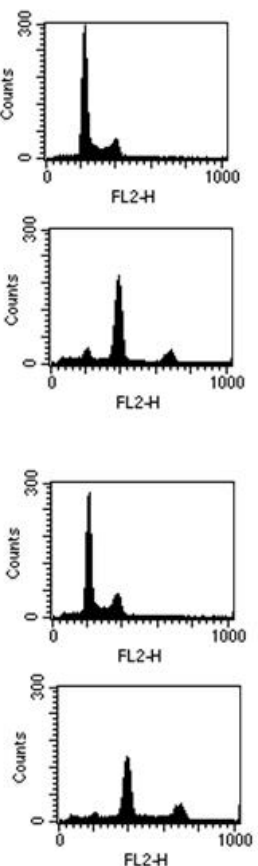

$24+24$

w/o
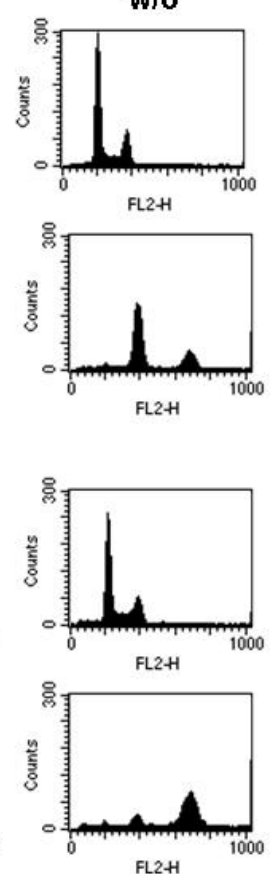

$24+48$

W/o
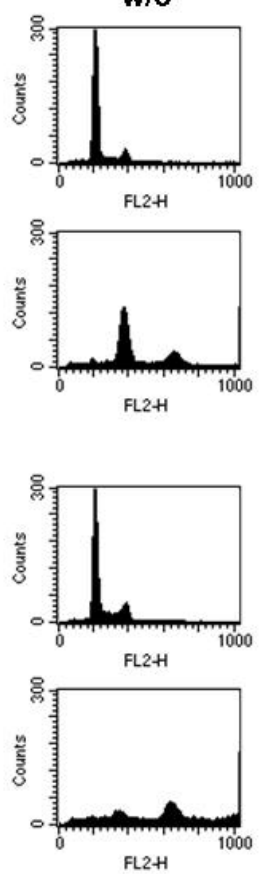

Figure 1: Cell cycle effect of Danusertib treatment on HCT116 cell lines wt and p53 -/-. HCT116 isogenic cell lines were treated with DMSO or $1 \mu$ M Danusertib for $6 \mathrm{hrs}, 24 \mathrm{hrs}, 24 \mathrm{hrs}$ after compound wash out $(24+24$ w/o $)$ or 48 hrs after compound wash out ( $24+48$ w/o), DNA content was analysed by flow cytometry as described in Material and Methods. 


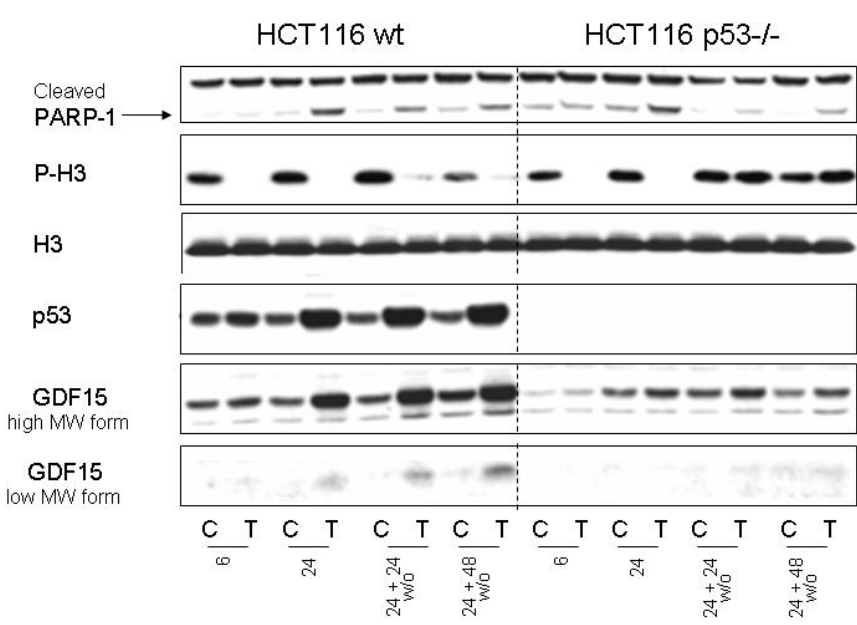

Figure 2: Effect of Danusertib treatment on the expression levels of some selected markers. HCT116 cells wt and p53 -/- were treated with DMSO (C) or $1 \mu \mathrm{M}$ Danusertib (T) for $6 \mathrm{hrs}$ and $24 \mathrm{hrs}$, then compound was wash out and cells kept for further $24 \mathrm{hrs}(24+24 \mathrm{w} / \mathrm{o})$ and $48 \mathrm{hrs}(24+48 \mathrm{w} / \mathrm{o})$. Then cells were collected at the different time points, lysed and analysed by Western Blotting for the expression of the several proteins as described in Material and Methods. Histone $\mathrm{H} 3$ was used as loading control.

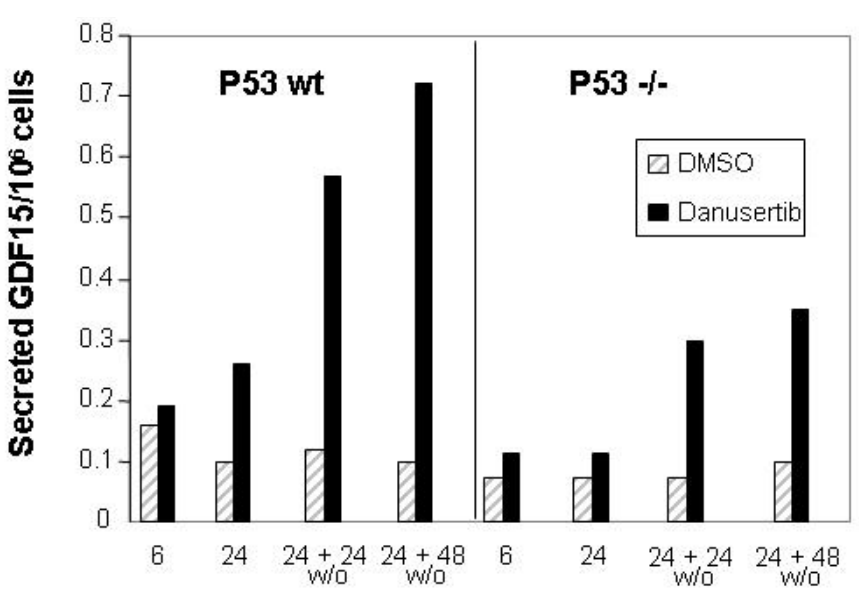

Time of treatment (hrs)

Figure 3: Levels of secreted GDF15 increase in the cell-free culture supernatants of cells treated with Danusertib. HCT116 wt and p53-/- were treated with DMSO (dashed bar) or $1 \mu \mathrm{M}$ Danusertib (black bar) for $6 \mathrm{hrs}$ and $24 \mathrm{hrs}$, then compound was washed out and cells kept for further $24 \mathrm{hrs}$ ( $24+$ $24 \mathrm{w} / \mathrm{o})$ and $48 \mathrm{hrs}(24+48 \mathrm{w} / \mathrm{o})$. Cell-free culture supernatants were removed at the different time points and assayed for secreted GDF15 by ELISA as described in Material and Methods.

homologue. Moreover, the human GDF15 levels in the plasma were normalized to the tumor size over the course of the study to exclude that the increase was only due to an increase in tumor volume, since it is known that plasma hGDF15 levels clearly correlate with increases in tumor xenograft volumes [27]. As shown in the inserted graph, the effect of Danusertib leads to tumor growth regression already $48 \mathrm{hrs}$ after the treatment whereas an increase in GDF15 levels is observed at this time point, indicating a drug induced effect.

In support of these findings, tumor tissues from the same animals were tested to confirm the modulation of some other well known markers for the compound activity. By Western Blotting, a clear up- regulation of both p53 and p21 proteins was observed starting from 24 hours after treatment and it was maintained up to 48 hours (Figure 5A). As expected a decrease of histone $\mathrm{H} 3$ phosphorylation was observed at 8 hours since Aurora- $\mathrm{B}$ is the mitotic kinase responsible for the direct phosphorylation of histone $\mathrm{H} 3$ in Serine 10 [35].

Immunohistochemistry analysis of the same tumors confirmed these observations for all the evaluated markers p53, p21 and p-histone H3 (Figure 5B). In tumor sections from mice treated with Danusertib, the number of cells positive for p53 and its downstream target, p21, significantly increased after $24-48$ hours as compared to the vehicletreated tumors ( $\mathrm{p}$-value in the range 0.015-0.0079). As expected, a clear down regulation of histone $\mathrm{H} 3$ phosphorylation after 8 hours was observed. In Supplementary Figure 1 representative pictures of the staining with different markers in vehicle and Danusertib treated tumors, are reported.

\section{GDF15 level in patients treated with Danusertib}

To investigate the use of GDF15 as a potential biomarker for the treatment response, we examined the levels of this protein in plasma of some patients with advanced solid tumors enrolled in one of the phase I clinical trials where Danusertib was administered as a 24-hour infusion in a 14-day cycle [10]. GDF15 protein levels were determined by ELISA in plasma samples collected before and after treatment with Danusertib at different doses $\left(500 \mathrm{mg} / \mathrm{m}^{2}, \mathrm{n}=3,580 \mathrm{mg} / \mathrm{m}^{2}, \mathrm{n}=6\right.$, and $\left.650 \mathrm{mg} / \mathrm{m}^{2}, \mathrm{n}=6\right)$. The systemic exposure of Danusertib increased with the dose showing a mean peak plasma concentration, which reached $4.25 \pm 0.7 \mu \mathrm{M}$ and a mean half-life of $22.2 \pm 4.5$ hours at the highest dose. Although GDF15 plasma levels were variable and especially elevated in most of the pre-treatment samples as expected for patients with metastatic cancers, they were generally further increased after treatment with Danusertib up to $48 \mathrm{hrs}$ (in the range of 1-2.2 fold, with patient $\mathrm{C}$ considered as an outlier sample) followed by a decrease at the longer time ( $72 \mathrm{hrs})$, as reported in Figure 6. Noteworthy, in plasma this decrease of GDF15 levels clearly correlates with the decrease in Danusertib concentration levels. As reported in Supplementary Figure 2, plasma concentrations of the compound decreased rapidly after the

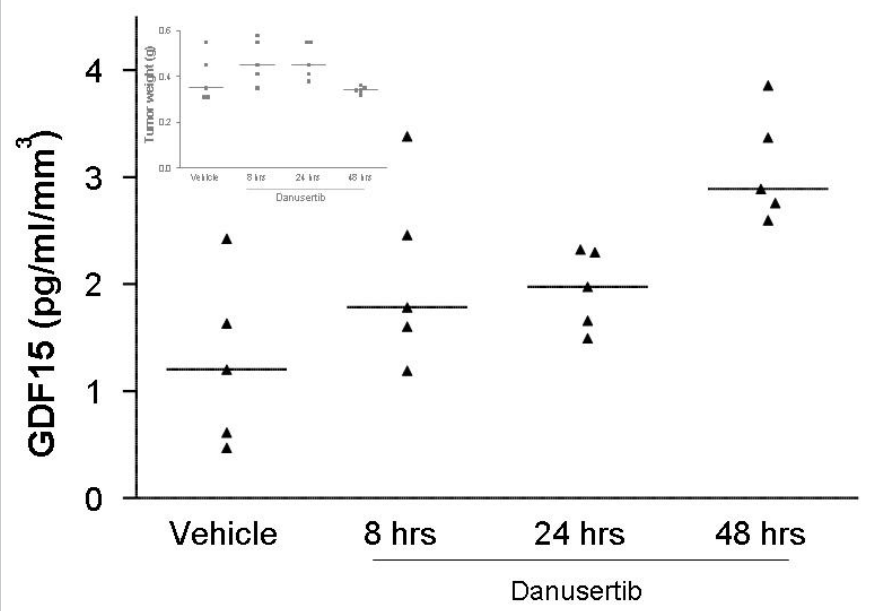

Figure 4: Increase of GDF15 plasma levels in mice treated with Danusertib. Mice bearing HCT116 tumor xenografts were treated with a single dose of Danusertib at $40 \mathrm{mg} / \mathrm{kg}$ bid i.v.. Determination of the GDF15 protein level in the plasma was measured by ELISA and normalized to tumor volume for each animal (5/each group). Bar, median of the group. Inserted graph: tumor weight for each animal in vehicle-treated ( $48 \mathrm{hrs}$ after the single treatment) and Danusertib treated animals at the different time points. 
A)

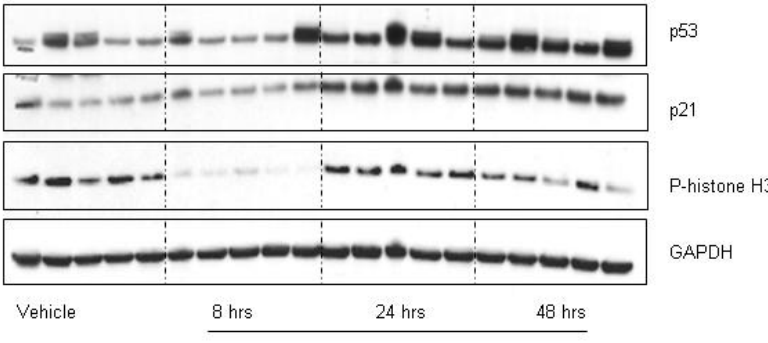

B)

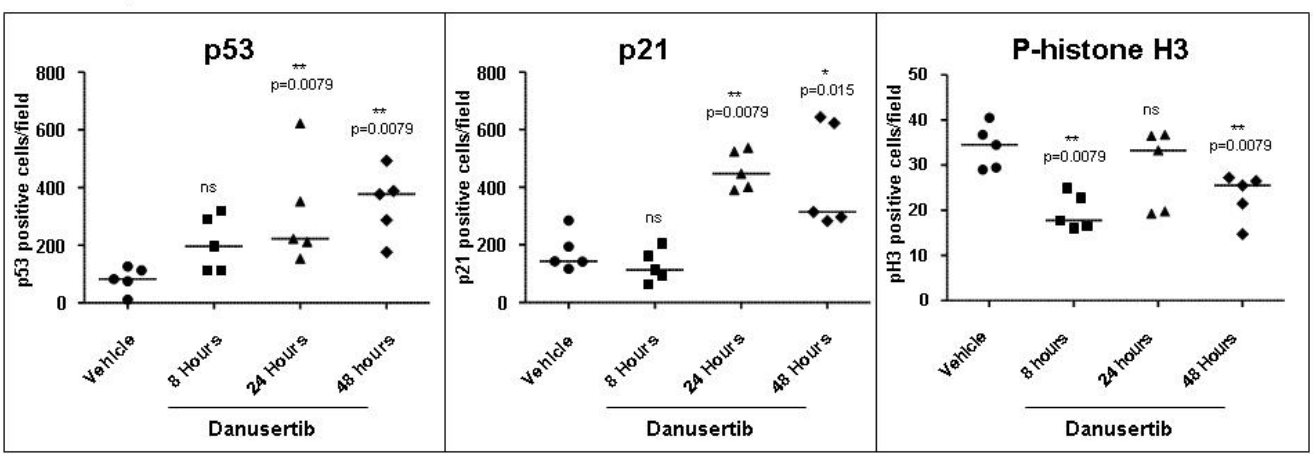

Figure 5: Evaluation of Danusertib activity in xenograft tumor. HCT116 tumor bearing mice were treated with vehicle or a single dose of Danusertib at 40 mg/ $\mathrm{kg}$ bid i.v. Animals (five for group) were sacrificed and tumors collected after 8,24 and 48 hrs and processed for the different analysis. A, Western blot analysis of phospho histone H3, p53, and p21 in tumor lysates. B, Immunohistochemistry analysis of the same tumors. Quantitative analysis of vehicle and Danusertib treated tumors immunostained with anti p53, anti p21 and anti phospho histone H3. Data are reported as the mean of positive cells counted in 4 viable fields at $200 x$ magnification for each sample. All mice per group were evaluated. Bar, median of the group.

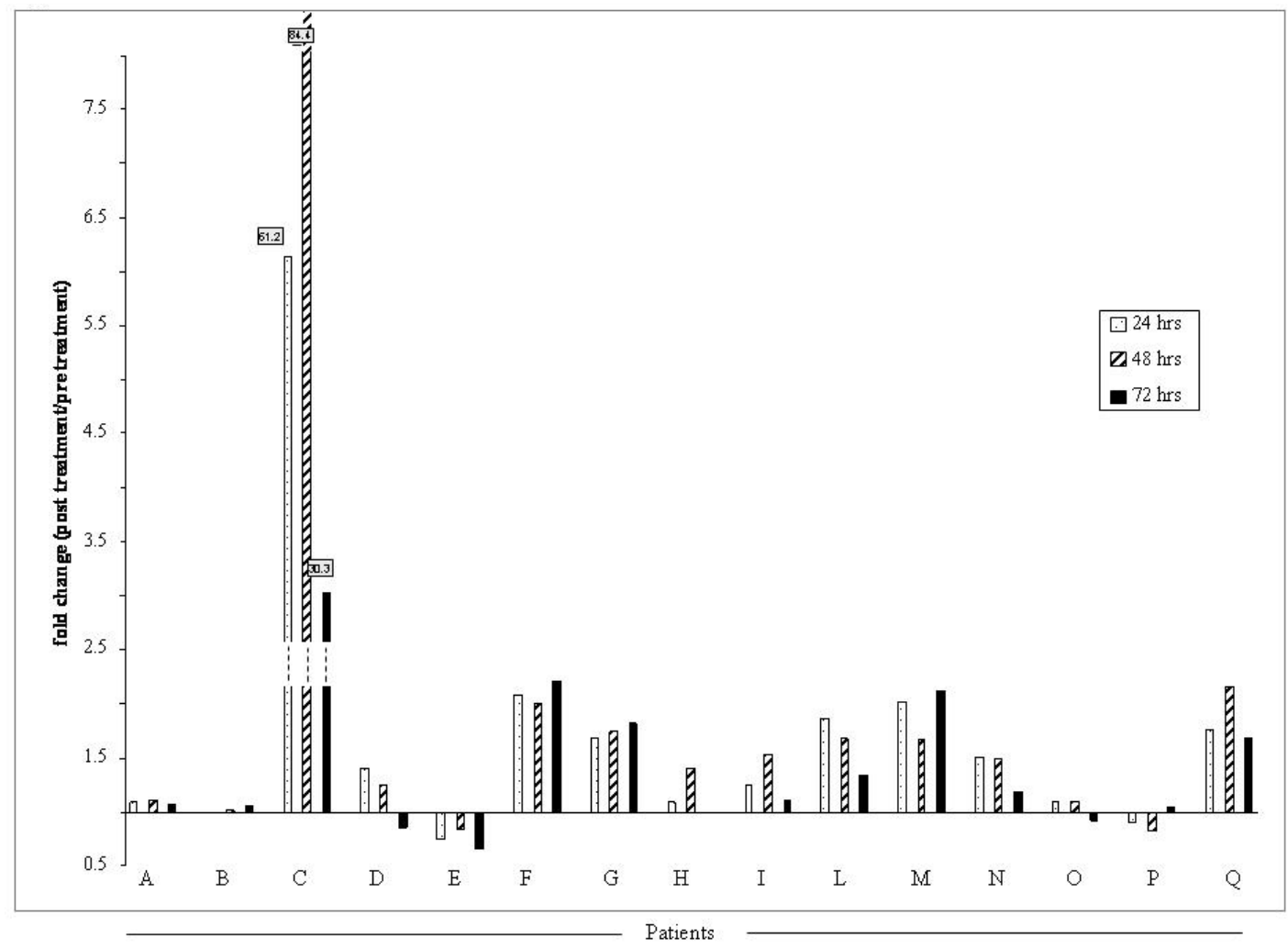

Figure 6: Plasma GDF15 levels in patients before and after treatment with Danusertib. Plasma samples from individual patients enrolled in the phase I clinical trial where Danusertib was administered as a 24-hour infusion in a 14-day cycle, were collected predose and after 24,48 and 72 hrs of the starting treatment. Data reported for some patients $(n=15)$ indicate the fold changes of the post-treatment versus pre-treatment samples for each analysed patient. 
end of treatment to reach a level of $0.076 \pm 0.047 \mu \mathrm{M}$ at $72 \mathrm{hrs}$ or below at a longer time $(0.012 \pm 0.007 \mu \mathrm{M}$ at $168 \mathrm{hrs})$. In these patients skin biopsies were also collected to assess biomarker modulation measured as a decrease in histone $\mathrm{H} 3$ phosphorylation. As reported previously, a decrease in phosphorylation of histone $\mathrm{H} 3$ was observed in the analysed samples, starting at $500 \mathrm{mg} / \mathrm{m}^{2}$ [10].These data indicate that the evaluation of GDF15 as a clinical biomarker of Danusertib activity is feasible in cancer patients.

\section{Discussion}

For the development of molecular therapeutic agents with specific cellular targets, it is crucial to understand the pharmacodynamic effects of these agents in cancer patients to guide clinical development. Danusertib, an Aurora kinase inhibitor, was shown to be active in a wide range of cancer cell lines and xenografts models [9]. In vitro as well as in vivo, Danusertib inhibits phosphorylation of histone $\mathrm{H} 3$ (Ser10), a protein implicated in chromosome condensation that is phosphorylated by Aurora B [35]. This effect was observed in skin, bone marrow, and xenografted tumors in mice, then translated into the clinic. Danusertib is currently undergoing phase I and II clinical trials for the treatment of advanced solid and hematological tumors [10-12]. In the phase II trials in solid tumors, the compound is being used either as a single agent or in combination. After a 24 hours infusion, Danusertib is well tolerated with target inhibition in skin at $\geq 500 \mathrm{mg} / \mathrm{m}^{2}$. Preliminary evidence of antitumor activity, including some partial responses and prolonged stable disease, were seen across a variety of advanced refractory cancers [10,12]. Predictive biomarkers or surrogate markers are still missing for Aurora kinase inhibitors and easy accessible plasma-based biomarkers would represent a significant advance. In an attempt to identify novel pharmacodynamic markers, in our previous work we sought to characterize at the transcriptional level the effects of Danusertib on different tumor cell lines, with wt or mutant p53 status (Bosotti R. and Carpinelli P., personal communication). We identified potential biomarkers for response to Danusertib treatment to monitor in p53 wt cells, including white blood cells which might be easily accessible to monitor gene or protein expression levels in a clinical setting. Among them, we selected GDF15, a secreted protein belonging to the transforming growth factor TGF- $\beta$ superfamily that has a role in regulating inflammatory and apoptotic pathways in injured tissues and during disease processes. GDF15 (also known as MIC1/PDF/ $\mathrm{PLAB} / \mathrm{Nag}-1 / \mathrm{PTGF} \beta$ ), is highly expressed in the placenta, and lower expression is found in epithelial cells from other organs including the prostate, colon, and brain [36,16]. Moreover, GDF15 is highly overexpressed in a wide variety of tumors such as those of the prostate, large bowel, and breast [37-41,24]. In low-grade prostate cancer, the degree to which GDF15 is bound to the extracellular matrix is the best independent predictor for the tumor progression, suggesting a role for GDF15 in its dissemination [25]. Either proapoptotic or antiapoptotic effects have been described for GDF15 dependent on the cellular context. For example, it has been shown that xenobiotic-induced and constitutive expression of GDF15 protein led to G1 or S phase cell cycle arrest as well as apoptosis in the cancer cells [42]. Although the mechanism of GDF15-mediated cell death is not clearly understood, several explanations have been reported. Evidences suggest that GDF15 can mediate $\mathrm{p} 53$-dependent growth suppression since the promoter of GDF15 has a potential regulatory site for p53 binding [43,21]. GDF15 was previously shown to be induced in a p53-dependent manner by treatment of different cell lines with several anticancer agents like genistein, retinoids, and resveratrol as well as chemotherapeutic drugs, including etoposide and doxorubicin $[21,23,24,27]$. For instance, the expression of endogenous GDF15 was remarkably induced by the chemotherapeutic drug etoposide in p53 wild-type cancer cell lines but not in p53-deficient cells $[19,17]$. However, GDF15 can also induce apoptotic death in cells deficient in p53, suggesting a p53-independent pathway for GDF15 activity. Moreover, in order to identify changes in gene expression after the first course of neoadjuvant chemotherapy for primary breast cancer, GDF15 was reported to be up-regulated in biopsies of patients after treatment with the combination of epirubicin and cyclophosphamide or epirubicin and taxol [44]. In the context of therapy, Huang et al. reported a molecular alteration of 51 unique genes in prostate carcinomas following chemotherapy with docetaxel and mitoxantrone and determined GDF15 expression to have increases ( $\geq 2$ fold) in $90 \%$ of the analysed samples [45]. Whether GDF15 induction could represent a tumor response gene, as the majority of subjects did have PSA reductions in the context of chemotherapy, or could represent an important modulator of resistance as there were no complete tumor responses in any of the subjects treated in this study, is not clear and needs further experimental studies. On the other hand, other transcription factors, such as the EGR-1 tumor suppressor protein, nuclear factor- $\kappa \mathrm{B}(\mathrm{NF}-\kappa \mathrm{B})$, and hypoxia-inducible factor-1 $\alpha$ (HIF-1 $\alpha$ ), can also induce GDF15 expression in certain cell types, including p53 wild-type and p53-mutant cancer cells [32,46,47]. Collectively, these studies indicate that the role and effect of GDF15 induction may be different depending on the cell type, stage and extent of the tumor producing it, as it has been found for other members of the TGF- $\beta$ superfamily. Plasma or serum markers have been very useful in the diagnosis of cancer. For example, detection of a keratin antigen in serum or plasma, such as tissue polypeptide antigen (TPA, fragments of CK8, 18 and 9), TPS (CK18 fragments and complexes) or CYFRA 21-1 (caspase cleaved CK19), has been viewed as a marker of bulk tumor burden while, some reports have suggested that they might also predict treatment outcome and overall survival $[48,49]$.

Tumor GDF15 can be readily detected by ELISA in the blood of mice with xenografted tumors and in cancer patients and therefore has been suggested to be used as a diagnostic tool for monitoring stage and extent of disease $[37,40,27]$. At present the precise role of GDF15 in tumor biology is unclear, but is likely that similar to TGF- $\beta$, its role will be different depending on the stage and type of the tumor producing it.

We identified GDF15 initially by microarray studies and then further validated its value by investigations in tissue cell culture, in mice plasma and finally in plasma of Danusertib treated patients. Taken together, these results suggest that serum levels of GDF15 could serve as surrogate biomarker for Danusertib activity which may translate to clinical utility. Considering that the primary objectives of the study were to determine the maximum-tolerated dose (MTD) and to define the dose-limiting toxicities (DLTs) during the first cycle of treatment with Danusertib, only a limited number of plasma samples were analysed. Future studies could examine the relevance of this and other genes identified in our previous study as surrogate biomarkers as well therapeutic targets to enhance drug efficacy.

\section{Acknowledgements}

The authors would like to thank R. Colombo and R. Alzani for critical reading of the manuscript

\section{References}

1. Frank R , Hargreaves R (2003) Clinical biomarkers in drug discovery and development. Nat Rev Drug Discov 2: 566-580.

2. Gruvberger SK, Ringner M, Eden P, Borg A, Ferno M, et al. (2003) Expression profiling to predict outcome in breast cancer: the influence of sample selection. Breast Cancer Res 5: 23-26. 
3. Nevins JR, Potti A (2007) Mining gene expression profiles: expression signatures as cancer phenotypes. Nat Rev Genet 8: 601-609.

4. Kastan MB (2007) Wild-type p53: tumors can't stand it. Cell 128: 837-840.

5. Vogelstein B, Lane D, Levine AJ (2000) Surfing the p53 network. Nature 408: 307-310

6. Bykov VJ, Issaeva N, Shilov A, Hultcrantz M, Pugacheva E, et al. (2002) Restoration of the tumor suppressor function to mutant $\mathrm{p} 53$ by a low-molecularweight compound. Nat Med 8: 282-288.

7. Roth JA (2006) Adenovirus p53 gene therapy. Expert Opin Biol Ther 6: 55-61.

8. Vassilev LT, Vu BT, Graves B, Carvajal D, Podlaski F, et al. (2004) In vivo activation of the p53 pathway by small-molecule antagonists of MDM2. Science303: 844-848.

9. Carpinelli P, Ceruti R, Giorgini ML, Cappella P, Gianellini L, et al. (2007) PHA739358, a potent inhibitor of Aurora kinases with a selective target inhibition profile relevant to cancer. Mol Cancer Ther 6: 3158-3168.

10. Cohen RB, Jones SF, Aggarwal C, von Mehren M, Cheng J, et al. (2009) A phase I dose-escalation study of danusertib (PHA-739358) administered as a 24-hour infusion with and without granulocyte colony-stimulating factor in a 14-day cycle in patients with advanced solid tumors. Clin Cancer Res15: 66946701.

11. Gontarewicz A, Brummendorf TH (2010) Danusertib (formerly PHA-739358)-a novel combined pan-Aurora kinases and third generation Bcr-Abl tyrosine kinase inhibitor. Recent Results Cancer Res184: 199-214.

12. Steeghs N, Eskens FA, Gelderblom H, Verweij J, Nortier JW, et al. (2009) Phase I pharmacokinetic and pharmacodynamic study of the aurora kinase inhibitor danusertib in patients with advanced or metastatic solid tumors. J Clin Oncol 27: 5094-5101.

13. Bootcov MR, Bauskin AR, Valenzuela SM, Moore AG, Bansal M, et al. (1997) MIC-1, a novel macrophage inhibitory cytokine, is a divergent member of the TGF-beta superfamily. Proc Natl Acad Sci U S A 94: 11514-11519.

14. Baek SJ, Kim KS, Nixon JB, Wilson LC, Eling TE (2001) Cyclooxygenase inhibitors regulate the expression of a TGF-beta superfamily member that has proapoptotic and antitumorigenic activities. Mol Pharmacol 59: 901-908.

15. Hromas R, Hufford M, Sutton J, Xu D, Li Y, et al. (1997) PLAB, a novel placental bone morphogenetic protein. Biochim Biophys Acta $1354: 40-44$.

16. Paralkar VM, Vail AL, Grasser WA, Brown TA, Xu H, et al. (1998) Cloning and characterization of a novel member of the transforming growth factor-beta/bone morphogenetic protein family. J Biol Chem 273: 13760-13767.

17. Tan M, Wang Y, Guan K, Sun Y (2000) PTGF-beta, a type beta transforming growth factor (TGF-beta) superfamily member, is a p53 target gene that inhibits tumor cell growth via TGF-beta signaling pathway. Proc Natl Acad Sci U S A 97: 109-114.

18. Bauskin AR, Brown DA, Kuffner $T$, Johnen $H$, Luo XW, et al. (2006) Role of macrophage inhibitory cytokine-1 in tumorigenesis and diagnosis of cancer. Cancer Res 66: 4983-4986.

19. Li PX, Wong J, Ayed A, Ngo D, Brade AM, et al. (2000) Placental transforming growth factor-beta is a downstream mediator of the growth arrest and apoptotic response of tumor cells to DNA damage and p53 overexpression. J Biol Chem275: 20127-20135

20. Ichikawa T, Suenaga Y, Koda T, Ozaki T, Nakagawara A (2008) TAp63dependent induction of growth differentiation factor 15 (GDF15) plays a critical role in the regulation of keratinocyte differentiation. Oncogene 27: 409-420.

21. Baek SJ, Wilson LC, Eling TE (2002) Resveratrol enhances the expression of non-steroidal anti-inflammatory drug-activated gene (NAG-1) by increasing the expression of p53. Carcinogenesis. 23: 425-434.

22. Kadara H, Schroeder CP, Lotan D, Pisano C , Lotan R (2006) Induction of GDF15/NAG-1/MIC-1 in human lung carcinoma cells by retinoid-related molecules and assessment of its role in apoptosis. Cancer Biol Ther 5: 518-522.

23. Lambert JR, Kelly JA, Shim M, Huffer WE, Nordeen SK, et al. (2006) Prostate derived factor in human prostate cancer cells: gene induction by vitamin $\mathrm{D}$ via a p53-dependent mechanism and inhibition of prostate cancer cell growth. J Cell Physiol 208: 566-574.

24. Wilson LC, Baek SJ, Call A, Eling TE (2003) Nonsteroidal anti-inflammatory drug-activated gene (NAG-1) is induced by genistein through the expression of p53 in colorectal cancer cells. Int J Cancer105: 747-753.
25. Bauskin AR, Brown DA, Junankar S, Rasiah KK, Eggleton S, et al. (2005) The propeptide mediates formation of stromal stores of PROMIC-1: role in determining prostate cancer outcome. Cancer Res 65: 2330-2336.

26. Yamaguchi K, Lee SH, Eling TE, Baek SJ (2004) Identification of nonsteroida anti-inflammatory drug-activated gene (NAG-1) as a novel downstream targe of phosphatidylinositol 3-kinase/AKT/GSK-3beta pathway. J Biol Chem 279: 49617-49623.

27. Yang H, Filipovic Z, Brown D, Breit SN, Vassilev LT(2003) Macrophage inhibitory cytokine-1: a novel biomarker for $\mathrm{p} 53$ pathway activation. Mol Cancer Ther 2: 1023-1029.

28. Bunz F, Dutriaux A, Lengauer C, Waldman T, Zhou S, et al. (1998) Requirement for p53 and p21 to sustain G2 arrest after DNA damage. Science 282: 14971501.

29. Soncini C, Carpinelli P, Gianellini L, Fancelli D, Vianello P, et al.(2006) PHA 680632 , a novel Aurora kinase inhibitor with potent antitumoral activity. Clin Cancer Res12: 4080-4089

30. Kastan MB, Kuerbitz SJ (1993) Control of G1 arrest after DNA damage. Environ Health Perspect 101 Suppl 5: 55-58.

31. Katayama H, Sasai K, Kawai H, Yuan ZM, Bondaruk J, et al. (2004 Phosphorylation by aurora kinase A induces Mdm2-mediated destabilization and inhibition of p53. Nat Genet 36: 55-62.

32. Baek SJ, Kim JS, Moore SM, Lee SH, Martinez J, et al. (2005) Cyclooxygenase inhibitors induce the expression of the tumor suppressor gene EGR-1 which results in the up-regulation of NAG-1, an antitumorigenic protein. Mo Pharmacol67: 356-364.

33. Baek SJ, Kim JS, Nixon JB, DiAugustine RP, Eling TE (2004) Expression of NAG-1, a transforming growth factor-beta superfamily member, by troglitazone requires the early growth response gene EGR-1. J Biol Chem279: 6883-6892.

34. Yoshioka H, Kamitani H, Watanabe T, Eling TE (2008) Nonsteroidal antiinflammatory drug-activated gene (NAG-1/GDF15) expression is increased by the histone deacetylase inhibitor trichostatin A. J Biol Chem 283: 33129-33137.

35. Crosio C, Fimia GM, Loury R, Kimura M, Okano Y, et al. (2002) Mitotic phosphorylation of histone $\mathrm{H} 3$ : spatio-temporal regulation by mammalian Aurora kinases. Mol Cell Biol 22: 874-885

36. Bottner M, Laaff M, Schechinger B, Rappold G, Unsicker K, et al. (1999) Characterization of the rat, mouse, and human genes of growth/differentiation factor-15/macrophage inhibiting cytokine-1 (GDF-15/MIC-1). Gene 237: 105111

37. Brown DA, Ward RL, Buckhaults P, Liu T, Romans KE, et al. (2003) MIC 1 serum level and genotype: associations with progress and prognosis of colorectal carcinoma. Clin Cancer Res9: 2642-2650.

38. Buckhaults $P$, Rago C, St Croix B, Romans KE, Saha S, et al. (2001) Secreted and cell surface genes expressed in benign and malignant colorectal tumors. Cancer Res 61: 6996-7001.

39. Karan D, Kelly DL, Rizzino A, Lin MF, Batra SK (2002) Expression profile of differentially-regulated genes during progression of androgen-independent growth in human prostate cancer cells. Carcinogenesis 23: 967-975.

40. Welsh JB, Sapinoso LM, Kern SG, Brown DA, Liu T, et al. (2003) Large-scale delineation of secreted protein biomarkers overexpressed in cancer tissue and serum. Proc Natl Acad Sci U S A 100: 3410-3415.

41. Welsh JB, Sapinoso LM, Su AI, Kern SG, Wang-Rodriguez J, et al. (2001) Analysis of gene expression identifies candidate markers and pharmacological targets in prostate cancer. Cancer Res 61: 5974-5978.

42. Agarwal MK, Hastak K, Jackson MW, Breit SN, Stark GR, et al. (2006) Macrophage inhibitory cytokine 1 mediates a p53-dependent protective arres in S phase in response to starvation for DNA precursors. Proc Natl Acad Sci U S A 103 : 16278-16283.

43. Albertoni M, Shaw PH, Nozaki M, Godard S, Tenan M, et al. (2002) Anoxia induces macrophage inhibitory cytokine-1 (MIC-1) in glioblastoma cells independently of p53 and HIF-1. Oncogene 21: 4212-4219.

44. Modlich O, Prisack HB, Munnes M, Audretsch W, Bojar H (2004) Immediate gene expression changes after the first course of neoadjuvant chemotherapy in patients with primary breast cancer disease. Clin Cancer Res 10: 6418-6431. 
Citation: Carpinelli P, Cappella P, Losa M, Croci V, Bosotti R, et al. (2011) GDF15 as a Novel Biomarker for Monitoring Danusertib Activity. J Mol Biomark Diagn S2:001. doi:10.4172/2155-9929.S2-001

45. Huang CY, Beer TM, Higano CS, True LD, Vessella R, et al. (2007) Molecular alterations in prostate carcinomas that associate with in vivo exposure to chemotherapy: identification of a cytoprotective mechanism involving growth differentiation factor 15. Clin Cancer Res13: 5825-5833.

46. Krieg AJ, Rankin EB, Chan D, Razorenova O, Fernandez S, et al. 2010) Regulation of the histone demethylase JMJD1A by hypoxia-inducible factor 1 alpha enhances hypoxic gene expression and tumor growth. Mol Cell Biol 30: $344-353$
47. Shim M, Eling TE (2005) Protein kinase C-dependent regulation of NAG-1/ placental bone morphogenic protein/MIC-1 expression in LNCaP prostate carcinoma cells. J Biol Chem 280: 18636-18642.

48. Barak V, Goike H, Panaretakis KW, Einarsson R (2004) Clinical utility of cytokeratins as tumor markers. Clin Biochem 37: 529-540.

49. Seregni E, Coli A, Mazzucca N (2004) Circulating tumour markers in breast cancer. Eur J Nucl Med Mol Imaging 31 Suppl 1: S15-22. 